\title{
Genetic Studies of Leptin Concentrations Implicate Leptin in the Regulation of Early Adiposity
}

Hanieh Yaghootkar,1,2,3 Yiying Zhang, ${ }^{4}$ Cassandra N. Spracklen,5,6 Tugce Karaderi,7,8,9,10 Lam Opal Huang, ${ }^{11}$ Jonathan Bradfield,12,13 Claudia Schurmann, ${ }^{14}$ Rebecca S. Fine, ${ }^{15,16,17}$ Michael H. Preuss, ${ }^{14}$ Zoltan Kutalik,,18,19 Laura B.L. Wittemans, ${ }^{7,20}$ Yingchang Lu, ${ }^{14,21}$ Sophia Metz, ${ }^{11}$ Sara M. Willems, ${ }^{20}$ Ruifang Li-Gao, ${ }^{22}$ Niels Grarup, ${ }^{11}$ Shuai Wang, ${ }^{23}$ Sophie Molnos, ${ }^{24,25}$ América A. Sandoval-Zárate, ${ }^{26}$ Mike A. Nalls, ${ }^{27,28}$ Leslie A. Lange, ${ }^{29}$ Jeffrey Haesser, ${ }^{30}$ Xiuqing Guo, ${ }^{31}$ Leo-Pekka Lyytikäinen,32,33 Mary F. Feitosa, ${ }^{34}$ Colleen M. Sitlani, ${ }^{35}$ Cristina Venturini, ${ }^{36}$ Anubha Mahajan,7,37 Tim Kacprowski, ${ }^{38,39}$ Carol A. Wang, ${ }^{23}$ Daniel I. Chasman, ${ }^{40,41}$ Najaf Amin, ${ }^{42}$ Linda Broer, ${ }^{43}$ Neil Robertson,7,37 Kristin L. Young, ${ }^{44}$ Matthew Allison, ${ }^{45}$ Paul L. Auer, ${ }^{46}$ Matthias Blüher, ${ }^{47}$ Judith B. Borja, ${ }^{48,49}$ Jette Bork-Jensen, ${ }^{11}$ Germán D. Carrasquilla, ${ }^{11}$ Paraskevi Christofidou, ${ }^{36}$ Ayse Demirkan, ${ }^{42}$ Claudia A. Doege, ${ }^{50}$ Melissa E. Garcia, ${ }^{51}$ Mariaelisa Graff, ${ }^{44,52}$ Kaiying Guo, ${ }^{4}$ Hakon Hakonarson, ${ }^{12,53}$ Jaeyoung Hong, ${ }^{23}$ Yii-Der Ida Chen, ${ }^{31}$ Rebecca Jackson, 54 Hermina Jakupović, ${ }^{11}$ Pekka Jousilahti, 55 Anne E. Justice,56 Mika Kähönen,57,58 Jorge R. Kizer,59,60 Jennifer Kriebel,24,25 Charles A. LeDuc,4 Jin Li,61 Lars Lind,62 Jian'an Luan,20 David A. Mackey,63 Massimo Mangino,36,64 Satu Männistö,55 Jayne F. Martin Carli, ${ }^{4}$ Carolina Medina-Gomez,42,43 Dennis O. Mook-Kanamori, ${ }^{22,65}$ Andrew P. Morris,7,66,67 Renée de Mutsert,22 Matthias Nauck,39,68 Ivana Prokic, ${ }^{42}$ Craig E. Pennell, ${ }^{23}$ Arund D. Pradhan, ${ }^{40,41}$ Bruce M. Psaty, ${ }^{69,70}$ Olli T. Raitakari, ${ }^{71,72,73}$ Robert A. Scott, ${ }^{20}$ Tea Skaaby, ${ }^{74}$ Konstantin Strauch, ${ }^{75,76}$ Kent D. Taylor, ${ }^{31}$ Alexander Teumer, ${ }^{39,77}$ Andre G. Uitterlinden, ${ }^{42,43}$ Ying Wu, ${ }^{5}$ Jie Yao, ${ }^{31}$ Mark Walker, ${ }^{78}$ Kari E. North, ${ }^{44}$ Peter Kovacs, ${ }^{47}$ M. Arfan Ikram, ${ }^{42,43}$ Cornelia M. van Duijn, ${ }^{42}$ Paul M. Ridker, ${ }^{40,41}$ Stephen Lye, ${ }^{79}$ Georg Homuth, ${ }^{38}$ Erik Ingelsson, ${ }^{61,80,81,82}$ Tim D. Spector, ${ }^{36}$ Barbara McKnight, 83 Michael A. Province,34 Terho Lehtimäki,32,33 Linda S. Adair,84 Jerome I. Rotter,31 Alexander P. Reiner, ${ }^{30}$ James G. Wilson, ${ }^{85}$ Tamara B. Harris, ${ }^{86}$ Samuli Ripatti, 17,26,87 Harald Grallert, ${ }^{24,25}$ James B. Meigs, $88,89,90$ Veikko Salomaa, 55 Torben Hansen,11 Ko Willems van Dijk,91,92,93 Nicholas J. Wareham, ${ }^{20}$ Struan F.A. Grant,12,53,94,95,96 Claudia Langenberg, ${ }^{20}$ Timothy M. Frayling, ${ }^{1}$ Cecilia M. Lindgren,7,17,97 Karen L. Mohlke,5 Rudolph L. Leibel,4 Ruth J.F. Loos, ${ }^{14,98}$ and

Tuomas O. Kilpeläinen ${ }^{11,99}$

Diabetes 2020;69:2806-2818 | https://doi.org/10.2337/db20-0070

${ }^{1}$ Genetics of Complex Traits, University of Exeter Medical School, Royal Devon \& Exeter Hospital, Exeter, U.K.

2Division of Medical Sciences, Department of Health Sciences, Luleå University of Technology, Luleå, Sweden

${ }^{3}$ Research Centre for Optimal Health, School of Life Sciences, University of Westminster, London, U.K.

4Division of Molecular Genetics, Department of Pediatrics, Columbia University, New York, NY ${ }^{5}$ Department of Genetics, University of North Carolina at Chapel Hill, Chapel Hill, NC ${ }^{6}$ Department of Biostatistics and Epidemiology, University of MassachusettsAmherst, Amherst, MA

${ }^{7}$ Wellcome Trust Centre for Human Genetics, University of Oxford, Oxford, U.K. ${ }^{8}$ Department of Biological Sciences, Faculty of Arts and Sciences, Eastern Mediterranean University, Famagusta, Cyprus

${ }^{9}$ Novo Nordisk Foundation Center for Protein Research, Faculty of Health and Medical Sciences, University of Copenhagen, Copenhagen, Denmark ${ }^{10}$ DTU Health Technology, Technical University of Denmark, Lyngby, Denmark ${ }^{11}$ Novo Nordisk Foundation Center for Basic Metabolic Research, Faculty of Health and Medical Sciences, University of Copenhagen, Copenhagen, Denmark
${ }^{12}$ Center for Applied Genomics, Division of Human Genetics, The Children's Hospital of Philadelphia, Philadelphia, PA

${ }^{13}$ Quantinuum Research LLC, San Diego, CA

${ }^{14}$ The Charles Bronfman Institute for Personalized Medicine, Icahn School of Medicine at Mount Sinai, New York, NY

${ }^{15}$ Department of Genetics, Harvard Medical School, Boston, MA

${ }^{16}$ Division of Endocrinology and Center for Basic and Translational Obesity Research, Boston Children's Hospital, Boston, MA

${ }^{17}$ Broad Institute of MIT and Harvard, Cambridge, MA

${ }^{18}$ Center for Primary Care and Public Health, University of Lausanne, Lausanne, Switzerland

${ }^{19}$ Swiss Institute of Bioinformatics, Lausanne, Switzerland

20MRC Epidemiology Unit, University of Cambridge, Cambridge, U.K.

${ }^{21}$ Division of Epidemiology, Department of Medicine, Vanderbilt-Ingram Cancer Center, and Vanderbilt Epidemiology Center, Vanderbilt University School of Medicine, Nashville, TN 
Leptin influences food intake by informing the brain about the status of body fat stores. Rare LEP mutations associated with congenital leptin deficiency cause severe early-onset obesity that can be mitigated by administering leptin. However, the role of genetic regulation of leptin in polygenic obesity remains poorly understood. We performed an exome-based analysis in up to 57,232 individuals of diverse ancestries to identify genetic variants that influence adiposity-adjusted leptin concentrations. We identify five novel variants, including four missense variants, in LEP, ZNF800, KLHL31, and ACTL9, and one intergenic variant near $K L F 14$. The missense variant Val94Met (rs17151919) in LEP was common in individuals of African ancestry only, and its association with lower leptin concentrations was specific to this ancestry $\left(P=2 \times 10^{-16}, n=3,901\right)$. Using in vitro analyses, we show that the Met94 allele decreases leptin secretion. We also show that the Met94 allele is associated with higher BMI in young African-ancestry children but not in adults, suggesting that leptin regulates early adiposity.

Leptin is an adipocyte-derived hormone that helps maintain homeostatic control of fat tissue mass by signaling the status of body energy stores to the appetite-regulating circuits of the brain (1). Rare homozygous mutations in the
${ }^{22}$ Department of Clinical Epidemiology, Leiden University Medical Center, Leiden, the Netherlands

23Department of Biostatistics, Boston University School of Public Health, Boston, MA

${ }^{24}$ German Center for Diabetes Research, München-Neuherberg, Germany

${ }^{25}$ Research Unit of Molecular Epidemiology, Institute of Epidemiology, Helmholtz Zentrum München Research Center for Environmental Health, München-Neuherberg, Germany

${ }^{26}$ Institute for Molecular Medicine Finland, Helsinki, Finland

${ }^{27}$ Laboratory of Neurogenetics, National Institute on Aging, National Institutes of Health, Bethesda, MD

28Data Tecnica International, Glen Echo, MD

${ }^{29}$ Division of Biomedical Informatics and Personalized Medicine, Department of Medicine, University of Colorado-Denver, Denver, C0

30Public Health Sciences Division, Fred Hutchinson Cancer Research Center, Seattle, WA

${ }^{31}$ The Institute for Translational Genomics and Population Sciences, Department of Pediatrics, The Lundquist Institute for Biomedical Innovation at Harbor-UCLA Medical Center, Torrance, CA

${ }^{32}$ Department of Clinical Chemistry, Fimlab Laboratories, Tampere, Finland

${ }^{33}$ Department of Clinical Chemistry, Finnish Cardiovascular Research Center Tampere, Faculty of Medicine and Health Technology, Tampere University, Tampere, Finland

${ }^{34}$ Division of Statistical Genomics, Department of Genetics, Washington University School of Medicine, St. Louis, M0

${ }^{35}$ Cardiovascular Health Research Unit, Department of Medicine, University of Washington, Seattle, WA

${ }^{36}$ Department of Twin Research and Genetic Epidemiology, Kings College London, London, U.K.

${ }^{37}$ Oxford Centre for Diabetes, Endocrinology and Metabolism, Radcliffe Department of Medicine, University of Oxford, Oxford, U.K.

${ }^{38}$ Department of Functional Genomics, Interfaculty Institute for Genetics and Functional Genomics, University Medicine Greifswald, Greifswald, Germany

${ }^{39}$ DZHK (German Center for Cardiovascular Research), partner site Greifswald, Greifswald, Germany

${ }^{40}$ Division of Preventive Medicine, Brigham and Women's Hospital, Boston, MA

${ }^{41}$ Harvard Medical School, Boston, MA

${ }^{42}$ Department of Epidemiology, Erasmus MC, University Medical Center Rotterdam, the Netherlands

43Department of Internal Medicine, Erasmus MC, University Medical Center Rotterdam, the Netherlands

${ }^{44}$ Department of Epidemiology, Gillings School of Global Public Health, University of North Carolina at Chapel Hill, Chapel Hill, NC

${ }^{45}$ Department of Family Medicine and Public Health, University of California, San Diego, La Jolla, CA
${ }^{46}$ Joseph J. Zilber School of Public Health, University of WisconsinMilwaukee, Milwaukee, WI

${ }^{47}$ Medical Department III - Endocrinology, Nephrology, Rheumatology, University of Leipzig Medical Center, Leipzig, Germany

${ }^{48}$ Office of Population Studies Foundation, Inc., Cebu City, Philippines

${ }^{49}$ Department of Nutrition and Dietetics, University of San Carlos, Cebu City, Philippines

${ }^{50}$ Department of Pathology and Cell Biology, Columbia University, New York, NY

${ }^{51}$ Laboratory of Epidemiology and Population Sciences, National Institute on Aging, Bethesda, MD

${ }^{52}$ Carolina Center for Genome Sciences, Chapel Hill, NC

53Department of Pediatrics, Perelman School of Medicine, University of Pennsylvania, Philadelphia, PA

${ }^{54}$ Division of Endocrinology, Diabetes, and Metabolism, Ohio State University, Columbus, $\mathrm{OH}$

${ }^{55}$ Department of Public Health Solutions, Finnish Institute for Health and Welfare, Helsinki, Finland

${ }^{56}$ Center for Biomedical and Translational Informatics, Geisinger, Danville, PA

${ }^{57}$ Department of Clinical Physiology, Tampere University Hospital, Tampere, Finland

${ }^{58}$ Department of Clinical Physiology, Finnish Cardiovascular Research Center Tampere, Faculty of Medicine and Health Technology, Tampere University, Tampere, Finland

${ }^{59}$ Cardiology Section, San Francisco Veterans Affairs Health Care System, University of Califomia San Francisco, San Francisco, CA

${ }^{60}$ Departments of Medicine and Epidemiology and Biostatistics, University of Califomia San Francisco, San Francisco, CA

${ }^{61}$ Division of Cardiovascular Medicine, Department of Medicine, Stanford University, Palo Alto, CA

${ }^{62}$ Department of Medical Sciences, Uppsala University, Uppsala, Sweden

${ }^{63}$ Centre for Ophthalmology and Visual Science, Lions Eye Institute, The University of Western Australia, Perth, West Australia, Australia

${ }^{64 N I H R ~ B i o m e d i c a l ~ R e s e a r c h ~ C e n t r e ~ a t ~ G u y ' s ~ a n d ~ S t ~ T h o m a s ' ~ F o u n d a t i o n ~ T r u s t, ~ L o n d o n, ~}$ U.K.

${ }^{65}$ Department of Public Health and Primary Care, Leiden University Medical Center, Leiden, the Netherlands

${ }^{66}$ Department of Biostatistics, University of Liverpool, Liverpool, U.K.

${ }^{67}$ Division of Musculoskeletal and Dermatological Sciences, University of Manchester, Manchester, U.K.

${ }^{68}$ Institute of Clinical Chemistry and Laboratory Medicine, University Medicine Greifswald, Greifswald, Germany

${ }^{69}$ Cardiovascular Health Research Unit, Departments of Epidemiology, Medicine, and Health Services, University of Washington, Seattle, WA

${ }^{70}$ Kaiser Permanente Washington Health Research Institute, Seattle, WA

${ }^{71}$ Centre for Population Health Research, University of Turku and Turku University Hospital, Turku, Finland

${ }^{72}$ Department of Clinical Physiology and Nuclear Medicine, Turku University Hospital, Turku, Finland 
leptin (LEP) gene can cause complete leptin deficiency that results in hyperphagia and severe early-onset obesity, which can be treated effectively by exogenous leptin administration $(2,3)$. Mice and patients heterozygous for these mutations show partial leptin deficiency and increased body weight (4-6).

In the general population, leptin concentrations correlate closely with body fat mass. However, there is wide interindividual variability; $\sim 10-20 \%$ of obese individuals have leptin concentrations that are similar to those observed in nonobese individuals, which is in part due to genetic differences $(7,8)$. Twin and family studies suggest that $30-50 \%$ of variation in leptin at any given level of adiposity and across different ethnic groups is explained by genetic differences (8). The implications of this variability for body weight regulation remain poorly understood.

Identification of genetic variants associated with circulating leptin may shed new light on the role of variability in leptin levels in the general population. In a recent genomewide association study (GWAS) of leptin concentrations, we identified four loci associated with leptin concentrations independent of BMI (9). The variant most strongly associated with leptin concentrations was rs10487505, located $21 \mathrm{~kb}$ upstream from $L E P$, in a region shown to harbor a long noncoding RNA (EST EL947753) that influences the transcriptional control of leptin expression (10). The leptin-decreasing allele of rs 10487505 was nominally associated with $\sim 0.03 \mathrm{~kg} / \mathrm{m}^{2}$ higher BMI in adults and 1.05-fold increased risk of early-onset obesity (9). More recently, the association of the leptin-decreasing allele of rs10487505 with higher adult BMI, body fat percentage, and risk of extreme obesity was replicated in the UK Biobank (10). The most pronounced association, however, was observed for body size at 10 years of age; carriers of the leptin-decreasing allele reported being "plumper" at age 10 years (compared with peers) more frequently than carriers of the allele associated with higher leptin concentration. The association between rs10487505 and childhood body size was recently replicated in 14,521 Norwegian children, and the peak effect of rs10487505 on BMI was observed in 1.5-year-old children (11).

In the current study, we sought to elucidate the genetic basis of leptin concentrations through screening genetic variants with an exome-targeted array in up to 57,232
73Research Centre of Applied and Preventive Cardiovascular Medicine, Turku University Hospital, Turku, Finland

${ }^{74}$ Center for Clinical Research and Disease Prevention, Bispebjerg and Frederiksberg Hospital, Copenhagen, Denmark

75Institute of Genetic Epidemiology, Helmholtz Zentrum München - German Research Center for Environmental Health, Neuherberg, Germany

${ }^{76}$ Chair of Genetic Epidemiology, Insitute of Medical Information Processing, Biometry, and Epidemiology (IBE), Faculty of Medicine, Ludwig Maximilian University Munich, München, Germany

${ }^{77}$ Institute for Community Medicine, University Medicine Greifswald, Greifswald, Germany

${ }^{78}$ Institute of Cellular Medicine (Diabetes), Newcastle University, Newcastle upon Tyne, U.K.

${ }^{79}$ Lunenfeld-Tanenbaum Research Institute, Mount Sinai Hospital, Toronto, Ontario, Canada

${ }^{80}$ Stanford Cardiovascular Institute, Stanford University School of Medicine, Palo Alto, CA

${ }^{81}$ Stanford Diabetes Research Center, Stanford University, Stanford, CA

${ }^{82}$ Molecular Epidemiology and Science for Life Laboratory, Department of Medical Sciences, Uppsala University, Uppsala, Sweden

${ }^{83}$ Department of Biostatistics, University of Washington, Seattle, WA

${ }^{84}$ Carolina Population Center, University of North Carolina at Chapel Hill, Chapel Hill, NC

${ }^{85}$ Department of Physiology and Biophysics, University of Mississippi Medical Center, Jackson, MS

${ }^{86}$ Laboratory of Epidemiology and Population Sciences, National Institute on Aging, National Institutes of Health, Bethesda, MD

${ }^{87}$ Public Health, University of Helsinki, Helsinki, Finland

${ }^{88}$ Division of General Internal Medicine, Massachusetts General Hospital, Boston, MA

${ }^{89}$ Department of Medicine, Harvard Medical School, Boston, MA

90Program in Population and Medical Genetics, Broad Institute of MIT and Harvard, Cambridge, MA

${ }^{91}$ Division of Endocrinology, Department of Internal Medicine, Leiden University Medical Center, Leiden, the Netherlands

${ }^{92}$ Einthoven Laboratory for Experimental Vascular Medicine, Leiden, the Netherlands
93Department of Human Genetics, Leiden University Medical Center, Leiden, the Netherlands

${ }^{94}$ Center for Spatial and Functional Genomics, Division of Human Genetics, The Children's Hospital of Philadelphia, Philadelphia, PA

${ }^{95}$ Division of Endocrinology and Diabetes, The Children's Hospital of Philadelphia, Philadelphia, PA

${ }^{96}$ Institute of Diabetes, Obesity and Metabolism, Perelman School of Medicine, University of Pennsylvania, Philadelphia, PA

${ }^{97} \mathrm{Big}$ Data Institute, Nuffield Department of Medicine, University of Oxford, Oxford, U.K.

${ }^{98}$ The Mindich Child Health and Development Institute, Icahn School of Medicine at Mount Sinai, New York, NY

99Department of Environmental Medicine and Public Health, Icahn School of Medicine at Mount Sinai, New York, NY

Corresponding authors: Hanieh Yaghootkar, h.yaghootkar@exeter.ac.uk, and Tuomas 0. Kilpeläinen, tuomas.kilpelainen@sund.ku.dk

Received 23 January 2020 and accepted 9 September 2020

This article contains supplementary material online at https://doi.org/10.2337/ figshare.12933737.

H.Y., Y.Z., R.J.F.L., and T.O.K. contributed equally to this study.

L.O.H. is currently affiliated with Steno Diabetes Center Copenhagen, Gentofte, Denmark. R.S.F. is currently affiliated with Vertex Pharmaceuticals Incorporated, Boston, MA. M.E.G. is currently affiliated with the National Heart, Lung, and Blood Institute (NHLBI), Bethesda, MD.

This work was conducted prior to M.E.G.'s current affiliation with the NHLBI, and, as such, the views expressed in this article do not represent the views of the $\mathrm{NHLBI}$, National Institutes of Health, or other government entity.

(C) 2020 by the American Diabetes Association. Readers may use this article as long as the work is properly cited, the use is educational and not for profit, and the work is not altered. More information is available at https://www.diabetesjournals .org/content/license. 
individuals of European, African, East Asian, or Hispanic ancestry. We confirm five previously established and identify five novel variants associated with leptin concentrations, including four missense variants in $L E P, Z N F 800$, KLHL31, and ACTL9, and one intergenic variant near KLF14. The novel LEP variant, Val94Met (rs17151919), is associated with leptin concentrations in adults of African ancestry only. The leptin-lowering Met94 allele of the rs17151919 variant is associated with higher BMI in young children but shows a weak or no association with BMI in adulthood, suggesting that leptin regulates early adiposity.

\section{RESEARCH DESIGN AND METHODS}

\section{Study Design}

We performed an exome-based association study using data from 35 cohorts comprising up to 57,232 adults ( $\geq 18$ years old), of whom 50,321 were of European descent, 4,387 of African descent, 2,036 of East Asian descent, and 488 of Hispanic descent. We carried out additional analyses in men and women separately. All analyses were performed in models combining studies of all ancestries and in European ancestry cohorts only, for both additive and recessive genetic models. All participating institutions and coordinating centers approved the project. Informed consent was obtained from all study participants. We report the study-specific design, sample quality control, and descriptive statistics in Supplementary Tables 1 and 2.

\section{Outcome Traits}

The participating studies acquired residuals for leptin concentrations (in $\mathrm{ng} / \mathrm{mL}$ ) using linear regression, adjusting for age, genome-wide principal components, and any study-specific covariates (e.g., study center). The residuals were calculated with and without adjustment for BMI. Studies with unrelated individuals acquired the residuals in men and women separately, whereas family-based studies additionally acquired sex-combined residuals with adjustment for sex as a covariate. Case-control studies acquired the residuals in case and control subjects separately. Finally, we rank transformed the residuals using inverse normal transformation to follow a distribution with a mean of 0 and an SD of 1 .

\section{Genotyping}

All participating studies performed genotyping using the Illumina HumanExome BeadChip. The genotype calling was performed using the designated manufacturer's software, followed by zCall. Study-specific quality control measures were implemented before the association analyses to remove poorly genotyped variants (Supplementary Table 3 ).

\section{Study-Level Association Analyses}

Associations of the exome-wide variants with the residuals of leptin concentrations were examined using linear mixed models implemented in either RAREMETALWORKER (12) or RVTESTS (13) (Supplementary Table 3). The model accounted for potential cryptic relatedness by incorporating a kinship matrix. We performed the single variant association analyses using both additive and recessive genotypic models. We also calculated covariance matrices capturing linkage disequilibrium (LD) relationships between markers within $1 \mathrm{Mb}$ for use in gene-level meta-analyses.

\section{Quality Control of Study-Level Association Results}

We applied the EasyQC package in $\mathrm{R}$ to association summary statistics from each participating study to identify cohort-specific quality control issues. This included 1) identifying issues with calculation of leptin residuals and transformation of the residuals, 2) identifying strand issues by comparing allele frequencies against reference alleles from the 1000 Genomes Project phase 1, and 3) identifying issues arising from population stratification.

\section{Single-Variant Meta-analyses}

The meta-analyses of summary statistics from the participating studies were carried out with use of RAREMETAL (14) by two different analysts in parallel. We excluded all variants with a call rate $<98 \%$, Hardy-Weinberg equilibrium $P$ value $<1 \times 10^{-6}$, or an allele frequency that strongly deviated from the 1000 Genomes reference frequency ( $>0.60$ for all-ancestry analyses and $>0.30$ for ancestry-specific analyses). To identify the leptin-associated variants, we used the array-wide Bonferroni-corrected threshold of $P<2 \times 10^{-7}$ for $\sim 250,000$ variants in the single variant analyses.

\section{Gene-Based Meta-analyses}

We performed gene-based analyses using the sequence kernel association test (15) (SKAT) and variable threshold (16) (VT) methods in RAREMETAL. The analyses were performed with two different sets of criteria (broad and strict) to select predicted damaging rare and low-frequency variants with minor allele frequency $(\mathrm{MAF})<5 \%$ annotated using five prediction algorithms: PolyPhen-2, HumDiv, HumVar, LRT, MutationTaster, and SIFT. The broad gene-based tests included nonsense, stop-loss, splice-site, and missense variants that were annotated as damaging by at least one of the five algorithms, whereas the strict tests only included variants predicted as damaging by all of the five algorithms. The statistical significance for the gene-based tests was set at a Bonferroni-corrected threshold of $P<$ $2.5 \times 10^{-6}$ for 20,000 genes.

\section{Age-Stratified BMI Analyses of Variants in and Near LEP}

To study the influence of age on the association of the Val94Met variant in LEP and the rs10487505 variant near LEP with childhood BMI, we performed age-stratified analyses in children with African and European ancestry from the Center for Applied Genomics at The Children's Hospital of Philadelphia (CHOP) cohort recruited from 2006 to present (17). The participants had multiple BMI measurements at different ages, and analyses were performed with measurements in 1-year age bins. The number of BMI measurements in each age bin is shown in Supplementary Tables 9 and 10. Statistical significance was 
defined as $P<0.05$. The Val94Met and rs10487505 variants were genotyped with the Illumina Infinium II HumanHap550 and Human610-Quad BeadChip and imputed to the HRC r1.1 reference panel with use of the Sanger Imputation Server. All participants were biologically unrelated, aged between 2 and 18 years, and between -3 and +3 SDs of corrected BMI using Centers for Disease Control and Prevention $\mathrm{z}$ scores. The study was approved by the institutional review board of CHOP. Parental informed consent was given for each study participant.

Additionally, we used information on comparative body size at age 10 years (data field 1,687) for 452,264 individuals of European ancestry and 8,154 individuals of African ancestry from the UK Biobank. The participants were asked to choose one of the three categories of "about average," "thinner," or "plumper" to describe their body size compared with average when they were 10 years old.

\section{Pathway Enrichment Analyses}

We used the ExomeChip-Data-driven Expression-Prioritized Integration for Complex Traits (EC-DEPICT) $(18,19)$ gene set enrichment analysis method to evaluate nonsynonymous index variants (strongest nonsynonymous variant within $\pm 1 \mathrm{Mb}$ boundary) with $P<5 \times 10^{-4}$ for association with either 1) leptin unadjusted for BMI or 2) leptin adjusted for BMI. EC-DEPICT"s primary innovation is the use of "reconstituted" gene sets, which consist of gene sets downloaded from several databases that have been extended based on publicly available large-scale coexpression data (18). Two analyses were performed: 1$)$ all coding variants $(N=93$ loci for leptin unadjusted for BMI and $N=91$ loci for leptin adjusted for BMI) and 2) coding variants with MAF $<5 \%$ only ( $N=77$ loci for leptin unadjusted for BMI and $N=65$ loci for leptin adjusted for BMI).

We also used PASCAL (pathway scoring algorithm) (20) to study the enrichment of exome-wide association results in gene sets and pathways with two estimation approaches: MAX and SUM. The MAX estimation is more powerful for single variant-driven associations, whereas the SUM estimation is more powerful when multiple variants are driving the signal (20). We used reconstituted gene sets from DEPICT and the reference data from UK10K (TwinsUK [21] and Avon Longitudinal Study of Parents and Children [ALSPAC] [22]) to estimate LD. The PASCAL analyses were performed for all exome-chip variants $\left(N_{\text {all }}=265,780\right.$ for leptin adjusted for BMI, $N_{\text {all }}=$ 265,780 for leptin unadjusted) and for coding variants only ( $N_{\text {coding }}=176,035$ for leptin adjusted for BMI, $N_{\text {coding }}=180,864$ for leptin unadjusted). No allele frequency or $P$ value thresholds were used to select variants for the PASCAL analyses. The pathway scoring method used by PASCAL combines individual gene scores without the need for a tuneable threshold parameter to determine inclusion of genes in the enrichment analysis (20).

Leptin adjusted for BMI is correlated with body fat-free mass (correlation with fat-free mass index [FFMI] in the
Fenland cohort $=-0.39$ ). The initial pathway analyses for leptin adjusted for BMI using EC-DEPICT and PASCAL suggested enrichment of skeletal muscle-related pathways. To make sure that the gene set enrichment results were not due to correlation between leptin adjusted for BMI and FFMI, we corrected the effect sizes using the following equation (23): $\beta_{\text {corrected }}=\beta_{\text {leptin }}-\left(\beta_{\mathrm{FFMI}} \times\right.$ $r_{\text {FFMIvs.LEPTIN }}$ ), where $r_{\text {FFMIvs.LEPTIN }}=-0.39$ (Pearson correlation coefficient in the Fenland Study). The $\beta_{\mathrm{FFMI}}$ coefficients were extracted from an ongoing exome-wide association study of FFMI in $\sim 500,000$ individuals.

\section{Collider Bias}

Given that we adjusted leptin concentrations for BMI in our exome-based analyses and leptin and BMI are strongly correlated ( $r=0.5-0.8$ ) (9), we tested all exome-based significant loci for evidence of collider bias (23-25). For each index, we extracted the association results from our BMI-unadjusted leptin analyses and from the largest published exome-wide analysis for BMI (19). We corrected BMI-adjusted associations for potential bias due to phenotypic correlation between leptin concentrations and BMI and compared the strength and significance of association with leptin concentrations unadjusted for BMI, leptin adjusted for BMI, and association with BMI (Supplementary Table 6).

\section{Expression Quantitative Trait Locus Colocalization Analyses}

The cis expression quantitative trait locus (eQTL) analyses were carried out by use of abdominal subcutaneous adipose tissue from 770 participants of the METSIM (METabolic Syndrome In Men) study, all of whom were Finnish men from Kuopio, Finland (26). The eQTL mapping in 770 METSIM individuals was performed by EPACTS implementing a linear mixed model to account for the population structure among the samples. The eQTLs were defined as cis (local) if the peak association was within $1 \mathrm{Mb}$ on either side of the exon boundaries of the gene. We also identified variants most strongly associated with genes/ transcripts from the index variant ("eSNP"). We used METSIM LD (based on $n=770$, HRC imputation) to assess LD $r^{2}$ between the index variant and the lead eSNP. If the pairwise LD was $r^{2}>0.80$, we performed a reciprocal conditional analysis. We tested association between the lead SNP and transcript level when the lead eSNP was included in the model and vice versa.

\section{Expression of the Potential Causal Genes in Preadipocytes and Mature Adipocytes}

We compared the expression of the candidate causal genes in the novel leptin-associated loci, including ZNF800, KLF14, KLHL31, ACTL9, CNTD1, and DNAJC18 in preadipocytes and mature adipocytes, two major constituent cell types of adipose tissue. Human preadipocytes isolated from adipose tissue were induced to undergo adipocyte differentiation in vitro (27). RNA samples were obtained from preadipocytes and lipid-laden mature adipocytes at postdifferentiation day 12 . 


\section{Impact of Val94Met Variant in LEP on Leptin Protein Stability}

We used UCSF Chimera 1.13.1 to model the threedimensional (3D) protein structure and valine-to-methionine substitution in the leptin protein (28). The Rotamers tool and the Dunbrack Rotamer Library were used to view and evaluate amino acid sidechain rotamers. The displayed orientation of methionine was chosen based on the clashes and contacts observed in the protein and hydrogen bonds (29). To predict protein stability, we used SDM (30), iStable (31), CUPSAT (32), and I-Mutant 2.0 (33). All analyses applied the 3D structure for leptin (identifier 1AX8) from the RSCB Protein Data Bank as the reference data set.

\section{Effect of the Val94Met Variant in LEP on Leptin Protein Stability and Secretion Rate}

We tested the effect of the Val94Met variant on leptin protein stability and secretion rate in human embryonic kidney (HEK)293 cells in vitro. Human leptin cDNA clone was obtained from Open Biosystems, Inc. (Huntsville, AL) and subcloned into pcDNA3.1 vector. The original cDNA clone encodes the Val94 variant. The 94Met variant was created using Quikchange II Site-Directed Mutagenesis Kit (Agilent Technologies, Santa Clara, CA), with the Val94 plasmid as template and the following mutagenesis primers: forward, 5'-atgccttccagaaacatgatccaaa tatccaac- $3^{\prime}$; reverse, $5^{\prime}$-gttggatatttggatcatgtttctggaaggcat- $\left.3^{\prime}\right)$. Plasmids carrying Val94 or $94 \mathrm{Met}$ cDNA $(0.05 \mu \mathrm{g})$ were introduced into HEK293 cells (0.65 million cells/well in 12-well plate) using Lipofectamine 2000 as previously described (34). For measurement of intracellular leptin protein turnover and secretion rates, cells were treated with protein synthesis inhibitor cycloheximide (CHX) $(20 \mu \mathrm{g} / \mathrm{mL})$ in fresh media for 0.5 and $1 \mathrm{~h}$ at $72 \mathrm{~h}$ posttransfection. Cells incubated with fresh media for $1 \mathrm{~h}$ without $\mathrm{CHX}$ were used as untreated controls. Conditioned media were saved for leptin assay, and cell lysate were prepared using NP-40 lysis buffer $(50 \mathrm{mmol} / \mathrm{L}$ Tris- $\mathrm{HCl}, \mathrm{pH} 8.0 ; 150 \mathrm{mmol} / \mathrm{L}$ $\mathrm{NaCl} ; 1 \mathrm{mmol} / \mathrm{L} \mathrm{DTT} ; 1 \mathrm{mmol} / \mathrm{L}$ EDTA; 0.5\% NP-40; $10 \%$ glycerol; and $1 \times$ Roche protein inhibitor mixture). Leptin concentrations in cell lysates and the amount of leptin in conditioned media were determined with a human leptin ELISA kit (R\&D Systems, Minneapolis, MN). Little or no cell debris was observed in the conditioned media after centrifugation, suggesting little or no cell breakage during the incubation. The experiments were carried out in duplicates or triplicates and repeated four times.

\section{Data and Resource Availability}

The meta-analysis summary results are available in the NHGRI-EBI GWAS Catalog.

\section{RESULTS}

Five Novel Genetic Variants Show Association With Leptin Concentrations Independent of Adiposity

To identify genetic variants associated with leptin concentrations, we tested the associations of 246,328 single nucleotide variants, genotyped on an exome-targeted genotyping array, with leptin concentrations in up to 57,232 individuals of European $(n=50,321)$, African $(n=$ $4,387)$, East Asian $(n=2,036)$, or Hispanic $(n=488)$ ancestry from 35 studies (Supplementary Tables 1 and 3). The exome array provides a detailed coverage of genecoding regions and includes tags for variants identified in previously published GWAS for human complex traits. Given the strong correlation between leptin and BMI ( $r=0.5-0.8)$ (9), we examined associations with leptin concentrations with and without adjustment for BMI. Additional analyses were performed in men $(n=23,862)$ and women $(n=$ $32,940)$ separately. All the analyses were performed in all ancestries combined and in European-ancestry individuals only.

We confirmed five previously established (9) and identified five novel variants associated with leptin concentrations. The novel associations include four missense variants, in LEP, ZNF800, KLHL31, and ACTL9, and one intergenic variant near KLF14 (Table 1 and Supplementary Table 4). The associations at already established loci include intergenic variants near LEP and CCNL1, a missense variant in GCKR, and intronic variants in COBLL1 and FTO (Table 1 and Supplementary Table 4). To detect additional independent signals at the 10 leptin-associated loci, we performed conditional analyses, but no further signals were identified.

The association between rs1121980 near FTO and leptin concentrations became nonsignificant after adjustment for BMI $\left(P_{\text {unadjusted }}=8 \times 10^{-17} ; P_{\text {BMI adjusted }}=\right.$ 0.45). The effects of all other known and novel loci were independent of BMI (Supplementary Table 5). We tested whether the adjustment for BMI, a strongly correlated covariate (23), may have introduced collider bias but found no evidence of such bias (Supplementary Table 6).

The strongest variant associated with leptin concentrations was rs791600, an intergenic variant near the LEP gene. The rs791600 variant is in LD (European [EUR] $r^{2}=0.70$ ) with the rs10487505 variant identified in our previously published GWAS (9), which is not included in the exome array and was therefore not available for analyses in the current study. In the prior GWAS, the rs10487505 variant showed a more significant association with BMI-adjusted leptin concentrations $\left(\beta=0.034\right.$ per allele, $P=2.7 \times 10^{-11}$, $n=29,252)$ than $r$ 791600 $(\beta=0.029$ per allele, $P=3.0 \times$ $10^{-9}, n=31,800$ ) and thus is still considered the lead variant at this locus (Supplementary Fig. 1).

Nine of the 10 identified loci showed an association with leptin concentrations in all ancestries combined and in European-ancestry-only analyses. However, the novel LEP variant Val94Met (rs17151919) only showed a significant association for all ancestries combined $(P=2 \times$ $10^{-16}$ ) and not in European-ancestry individuals alone $(P=0.47)$ (Supplementary Table 7). In further ancestrystratified analyses, we observed that the Met94-coding allele is common in populations of African ancestry (MAF $=8 \%$ ), less common in those with Hispanic ancestry $(\mathrm{MAF}=2 \%)$, very rare in those with European ancestry 


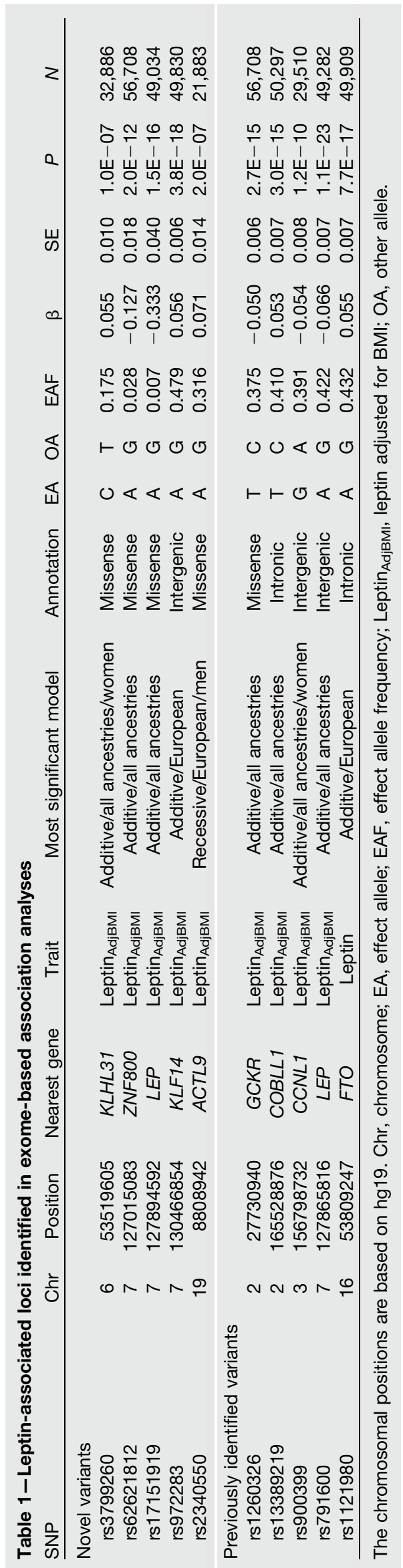


$(\mathrm{MAF}=0.02 \%)$, and monomorphic in people with East Asian ancestry (35). In individuals of African descent, each Met94-coding allele was associated with 0.34 SDs lower leptin concentrations $\left(P=2 \times 10^{-16}, n=3,901\right)$ (Supplementary Fig. 2). The direction of effect was consistent in individuals with Hispanic ( $-0.21 \mathrm{SD}$ effect per allele, $P=0.29, n=488)$ and individuals with European $(-0.19$ SD effect per allele, $P=0.47, n=44,401$ ) ancestry but did not reach statistical significance, most likely because very few carriers were available $\left(N_{\text {Hispanic }}=24, N_{\text {EUR }}=15\right)$ (Supplementary Table 7).

\section{Gene-Based Analysis Identifies Two Novel Genes With Sex-Specific Effect on Leptin}

In addition to single variant-based association tests, we performed gene-based tests using rare and low-frequency coding variants in aggregate $(15,16)$ (RESEARCH DESIGN AND METHODS). We identified two genes associated with leptin concentrations. CNTD1 showed association with leptin concentrations unadjusted for BMI in men $\left(P=1 \times 10^{-7}\right)$ but not in women $(P=0.27)$ (Table 2 and Supplementary Table 8 ). The association in men was driven by five coding variants and was strongly attenuated by adjustment for BMI $(P=$ 0.007), suggesting that the association of CNTD1 with leptin concentrations may be due to a link between CNTD1 and adiposity, although no such connection has previously been reported. The CNTD1 gene encodes cyclin N-terminal domain-containing 1 , which is critical for meiotic crossover maturation and deselection of excess precrossover sites.

Another gene, DNAJC18, showed association with BMIadjusted leptin concentrations in women $\left(P=6 \times 10^{-8}\right)$ but not men $(P=0.02)$. The association in women was driven by two coding variants (Table 2 and Supplementary Table 8). DNAJC18 is part of the DnaJ Hsp family. However, no function has yet been described to $\mathrm{C} 18$ subfamily.

\section{LEP Val94Met Regulates Leptin Secretion and Early Adiposity}

The Val94Met (rs17151919) LEP variant was associated with BMI-adjusted leptin concentrations in individuals of African ancestry. A previous study in 2,129 African Americans in the Coronary Artery Risk Development Study in Young Adults (CARDIA) (not included in the present metaanalyses) reported a significant association between the leptin-decreasing Met94 allele of the Val94Met (rs17151919) variant in $L E P$ and up to $1.12 \mathrm{~kg} / \mathrm{m}^{2}$ higher $\mathrm{BMI}$ in adulthood $(P=0.018)(36)$. However, results from two larger studies of adult BMI by the African Ancestry
Anthropometry Genetics Consortium ( $n=42,752, P=$ 0.88 ) (37) and the African ancestry population of the UK Biobank study $(N=7,820, P=0.17)$ did not replicate the association. Nevertheless, among the African ancestry population in the UK Biobank, carriers of the leptin-decreasing Met94 allele reported more often that at age 10 years they were "plumper" compared with peers (odds ratio $1.11, P=0.04$ ), suggesting that the effect of this variant may be age dependent. To study the influence of age, we performed age-stratified analyses in up to 2,726 children with African ancestry from the Center for Applied Genomics at $\mathrm{CHOP}$ cohort (17). Comparison of the effect sizes across different age points revealed that each leptin-decreasing Met94 allele was associated with $0.12-0.20$ units higher BMI $z$ score between the ages 3 and 7 years $(P<0.05)$. The most pronounced effect was reached at age 6 years (Fig. 1 and Supplementary Table 9), and no association with BMI was observed after age 8 years ( $\beta=-0.04$ to 0.05 ) (Fig. 1 and Supplementary Table 9), suggesting that the BMI-increasing effect of the Met94 allele wanes shortly before puberty. The rs10487575 variant near LEP showed a trajectory of association with childhood BMI similar to that of the Val94Met variant, but the effect sizes were much more modest (Fig. 1 and Supplementary Table 10), consistent with the fivefold smaller effect of rs 10487505 on leptin concentrations compared with Val94Met in adults (Table 1).

The Val94Met variant is located at position 94 in the 167 amino acid leptin precursor protein and results in a valineto-methionine change at position 73 of the mature protein (Fig. 2A). Position 73 is situated at the leptin protein surface and is not believed to be involved in binding of leptin to its receptor. Nevertheless, structural prediction tools (30-33) suggested that the substitution of valine with methionine at this position is likely to lead to reduced stability of the mature leptin protein (Fig. 2A, Supplementary Figs. 3 and 4, and Supplementary Table 11). This is consistent with our observation that the methioninecoding allele is associated with lower leptin concentrations.

To study the impact of the Val94Met variant on the intracellular turnover of the leptin protein and its secretion rate, we performed in vitro experiments in HEK293 cells. Leptin secretion rate-calculated as the amount of leptin secreted in $1 \mathrm{~h}$ normalized to the respective cellular leptin content-was 20.4\% lower in Met94 than in Val94 cells ( $P=0.0007$ by repeated-measures one-way ANOVA) $72 \mathrm{~h}$ posttransfection (Fig. $2 B$ ). Leptin secretion rates between 48 and $72 \mathrm{~h}$ posttransfection and during a 1-h

\begin{tabular}{|c|c|c|c|c|c|c|c|c|c|c|}
\hline Gene & Chr & Position & Trait & Most significant model & Method & $N$ & $P$ & $\beta$ & SE & $N$ variants \\
\hline CNTD1 & 17 & $\begin{array}{c}40950810- \\
40963605\end{array}$ & Leptin & $\begin{array}{l}\text { Additive/European/ } \\
\text { men }\end{array}$ & SKAT broad & 18,882 & $1.3 \mathrm{E}-07$ & 0.898 & 0.165 & 5 \\
\hline DNAJC18 & 5 & $\begin{array}{c}138743559- \\
138780898\end{array}$ & Leptin $_{\text {AdjBMI }}$ & $\begin{array}{c}\text { Additive/all ancestries/ } \\
\text { women }\end{array}$ & SKAT strict & 29,510 & $5.5 \mathrm{E}-08$ & 0.757 & 0.169 & 2 \\
\hline
\end{tabular}

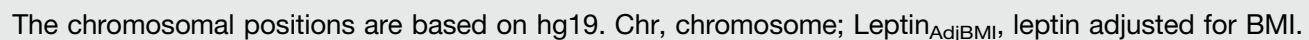



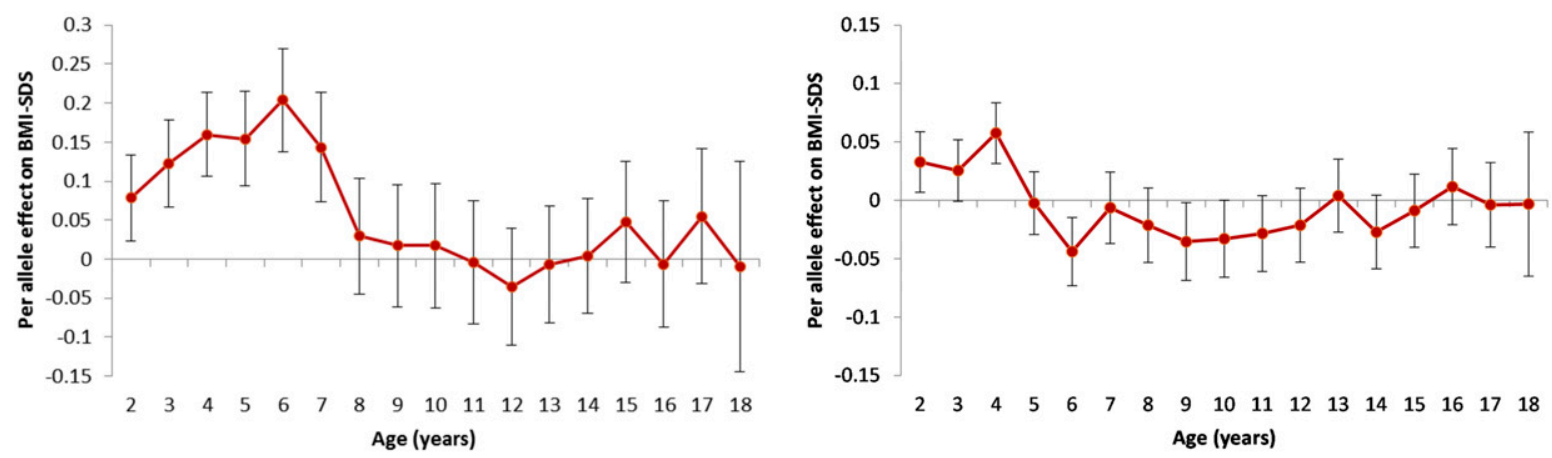

Figure 1-Association of the leptin-decreasing alleles of the LEP Val94Met (rs17151919) variant (on the left) and the rs10487505 variant near LEP (on the right) with BMI SD score (BMI-SDS) in the CHOP cohort. The analyses for the Val94Met variant were performed in up to 2,726 African-ancestry participants and the analyses for the rs 10487505 variant in up to 3,681 African- and European-ancestry participants of the $\mathrm{CHOP}$ cohort. The $y$-axis shows the effect of each leptin-decreasing allele on BMI at each age year. The error bars indicate 1 SEM.

treatment with CHX were $11.8 \%(P=0.0005)$ and $17.9 \%$ $(P=0.0002)$ lower, respectively, in Met94 compared with Val94 (Supplementary Fig. 5). Notably, no difference was found in the intracellular turnover rate of leptin between Val94 and Met94 cells during a 0.5- or 1.0-h incubation with CHX to impair protein synthesis (Fig. 2C). The unchanged turnover rate incorporates protein secretion and degradation, suggesting that decreased leptin secretion rate was likely associated with increased intracellular leptin degradation in Met94 cells. Overall, these in vitro experiments suggest that methionine substitution in position 73 of the mature leptin protein decreases the rate of leptin secretion from the cells, which may contribute to the association of the Met94 allele with lower leptin concentrations.

\section{ZNF800 Locus Regulates Adipose Gene Expression and Body Composition}

The Pro103Ser (rs62621812) variant in ZNF800 changes the amino acid sequence of $\mathrm{CH} 2$ zinc finger protein, a putative transcription factor (38). We found that the Ser103 allele (frequency 2.8\%) is associated with lower BMI-adjusted leptin concentrations $\left(P=2.0 \times 10^{-12}\right)$. As shown before (26), Pro103Ser is the lead variant associated with expression of ZNF800 in subcutaneous adipose tissue in the Finnish METSIM study $\left(P=2.4 \times 10^{-16}\right)$; the Ser103 allele is associated with higher ZNF800 expression levels (Supplementary Table 12). ZNF800 is a master regulator in subcutaneous adipose tissue, as the Pro103Ser variant has also been associated with adipose tissue expression of nine other genes (26). In the eQTL data, the leptin-decreasing Ser103 allele was not significantly associated with the expression of LEP $(P=0.20)$, located $866 \mathrm{~kb}$ downstream, and the observed direction of the effect on $L E P$ expression was opposite that observed for leptin concentrations ( $\beta=0.14 \mathrm{SD} /$ allele vs. $\beta=-0.13 \mathrm{SD} /$ allele, respectively), suggesting that the leptin-lowering effect of the Ser103 allele on leptin concentrations is unlikely to be mediated by direct transcriptional regulation of $L E P$.
In the UK Biobank study, we found that each leptindecreasing Ser103 allele is associated with $0.14 \mathrm{~kg} / \mathrm{m}^{2}$ higher BMI $\left(P=8.1 \times 10^{-6}\right)$. However, there was no association between Ser103 allele and body fat percentage $(-0.045 \%$ per allele, $P=0.25)$, indicating that the variant impacts BMI primarily by increasing fat-free body mass. Indeed, the leptin-decreasing Ser103 allele was associated with a $0.33 \mathrm{~kg}$ higher fat-free mass $\left(P=4.6 \times 10^{-20}\right)$ and only $0.13 \mathrm{~kg}$ higher fat mass $(P=0.023)$. The Ser103 allele is associated with higher expression of the ZNF800 gene in the tibial nerve (GTEx V8) $\left(P=1.4 \times 10^{-6}, n=532\right)$ that innervates the muscles of the leg and was previously identified for association with increased appendicular lean mass (39). There was no association between the Ser103 allele and self-reported body size at age 10 years $(P=$ 0.75).

\section{The KLF14 Locus Regulates Adipogenesis and Fat Distribution}

The rs972283 variant $\left(\mathrm{MAF}_{\mathrm{EUR}}=48 \%\right)$, associated with leptin concentrations, is located $51 \mathrm{~kb}$ upstream from KLF14 and $2.5 \mathrm{Mb}$ downstream from LEP and is in nearperfect LD with previously reported GWAS variants for type 2 diabetes (40), insulin resistance (41), HDL cholesterol (42), and body fat distribution (43). As previously reported (26), rs972283 is associated with KLF14 expression in subcutaneous adipose tissue (Supplementary Table 12). As KLF14 is a master regulator in adipose tissue, rs972283 is also associated with the expression of multiple other genes in trans (44). No significant association was observed between rs972283 and LEP expression in the METSIM eQTL study (44), suggesting that KLF14 may not regulate leptin production at the transcriptional level, at least not in men. Lower expression of KLF14 has been implicated in impaired adipogenesis due to defective adipocyte glucose uptake in women, characterized by the presence of fewer but larger adipocytes and a shift in fat distribution from gynoid stores to abdominal tissues (44). However, while the effects of KLF14 on adipogenesis 

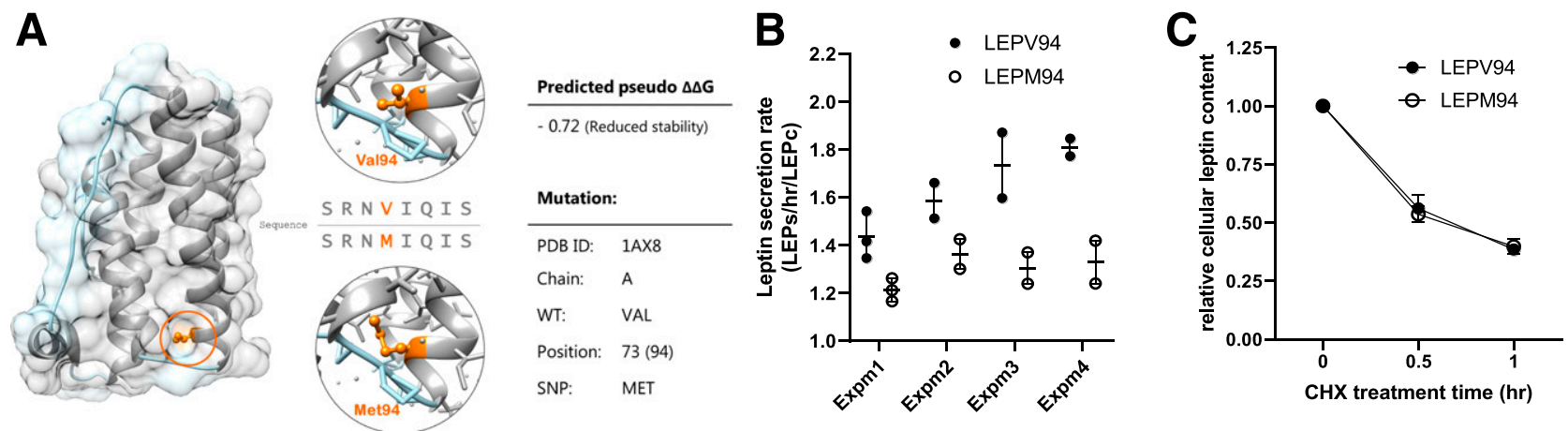

\begin{tabular}{|c|c|}
\hline LEPV94 & $1.62 \pm 0.18$ \\
\hline LEPM94 & $1.29 \pm 0.09$ \\
\hline $\mathrm{F}(1,8)$ & 28.82 \\
\hline $\mathrm{p}$ & 0.0007 \\
\hline
\end{tabular}

\begin{tabular}{|c|c|c|}
\hline & $0.5 \mathrm{hr}$ & $1 \mathrm{hr}$ \\
\hline LEPV94 & $0.56 \pm 0.06$ & $0.39 \pm 0.02$ \\
\hline LEPM94 & $0.54 \pm 0.03$ & $0.40 \pm 0.03$ \\
\hline $\mathrm{P}$ (V94 vs M94) & 0.316 & 0.366 \\
\hline
\end{tabular}

Figure 2-Impact of Val94Met transversion at LEP rs17151919 on leptin secretion rate in HEK293 cells. The rs17151919 variant changes valine to methionine in position 73 of the mature leptin protein. $A$ : The 3D illustration of leptin structure derived from RSCB Protein Data Bank (PDB) and modified with UCSF Chimera1.13.1. The prediction of protein stability is derived from the SDM server (30). B: Leptin secretion rates for Val94 and Met94 expressed as the amount of leptin secreted (ng) during a 1-h incubation (72-73 h posttransfection) (LEPs/h) normalized by the respective cellular leptin content (LEPC) in untreated control cells at the end of incubation. Individual data points from four separate experiments (each with two to three technical replicates) are plotted. The normality of data distribution was examined using D'Agostino and Pearson normality test ( $P=0.65$ and 0.54 for LEPV94 (Val94) and LEPM94 (Met94), respectively), and repeated-measures one-way ANOVA was performed to assess the difference in secretion rate between the genotypes. Mean $\pm \mathrm{SD}$ and AVOVA results (F and $P$ values) are reported in the table below the graph. C: Intracellular leptin turnover rates for Val94 and Met94 alleles, obtained by measuring the relative cellular leptin contents in the untreated control cells (defined as 1 for the respective LEP variant) and in samples treated with the protein synthesis inhibitor $\mathrm{CHX}(20 \mu \mathrm{g} / \mathrm{mL})$ for 0.5 and $1.0 \mathrm{~h}$. Mean $\pm \mathrm{SD}$ at each time point from four separate experiments (each with two to three technical replicates) is plotted. Paired $t$ test was used to assess the genotype effect on the fractions of cellular LEP that remained after $0.5 \mathrm{~h}$ and $1.0 \mathrm{~h}$ of $\mathrm{CHX}$ treatment ( $P$ values are reported in the table below the graph). The average hourly turnover rates for Val94 and Met94 were $61 \pm 2 \%$ and $60 \pm$ $3 \%$, respectively, calculated by subtraction of the percent cellular LEP that remained after $1 \mathrm{~h}$ of $\mathrm{CHX}$ treatment from those of the respective untreated controls (defined as 100\%). hr, hour(s); ID, identifier; M94, Met94; pseudo $\Delta \Delta \mathrm{G}$, predicted stability difference score; SNP, single nucleotide polymorphism; V94, Val94; WT, wild type.

and adipose redistribution have been found to be specific to women, there was no difference in the association of rs972283 with leptin levels between men and women (Supplementary Table 4).

Interestingly, the carriers of the rs972283-G allele more frequently reported being plumper $\left(P=2.8 \times 10^{-5}\right)$ and shorter $(P=0.014)$ than average at age 10 years in the UK Biobank than noncarriers, whereas the same allele was associated with a lower BMI $\left(P=6.8 \times 10^{-9}\right)$ and increased height $(P=0.010)$ in adults, suggesting that the effect of the rs 972283 variant on body size may change during life course. In previous GWAS of adults, the rs972283-G allele was identified as associated with higher risk of type 2 diabetes (40) and insulin resistance (41) and lower hip circumference (adjusted for BMI) (43) and HDL cholesterol (42). In the UK Biobank study, the rs972283-G allele was associated with lower body fat percentage in adults $\left(P=5.9 \times 10^{-22}\right)$.

\section{The KLHL31 Locus Is Implicated in Adipogenesis in Adult Females}

The Val156Ile (rs3799260) variant $\left(\mathrm{MAF}_{\mathrm{EUR}}=18 \%\right)$ in KLHL31, associated with leptin concentrations in femaleonly analyses, changes the amino acid sequence of the Kelch-like family member 31 protein. KLHL31 suppresses Wnt- $\beta$-catenin signaling that is involved in promoting adipocyte differentiation and suppressing oxidative metabolism in adipocytes. The Val156Ile variant is predicted to be benign/tolerated by SIFT/PolyPhen $(45,46)$. Previous genetic associations have identified a variant in low LD (rs7739232) (EUR $\left.r^{2}=0.27\right)$ to be associated with BMIadjusted hip circumference, also specific to women (43). The rs7739232 variant was not included in the exome array and was thus not analyzed in the current study. Our in vitro experiments showed that KLHL31 is only expressed in mature adipocytes, but not in preadipocytes (Supplementary Fig. 6), suggesting that the gene is developmentally regulated.

In the UK Biobank, similar to the variants in and near $L E P$, the leptin-decreasing Ile156 allele carriers more often reported being plumper than average at age 10 years $(P=$ $\left.5.6 \times 10^{-6}\right)$, but there was a weaker association with higher BMI $(P=0.045)$ in adulthood.

\section{In Men, the ACTL9 Locus May Regulate Leptin Concentrations in a Cell-Nonautonomous Fashion}

Homozygosity for the minor allele of the Ser37Phe (rs2340550) variant in ACTL9 was associated with leptin concentrations in men only in a recessive genetic model. While the Ser37Phe variant is predicted to be benign/ tolerated by SIFT/PolyPhen, another missense variant, Ala51Val (rs10410943), in high LD (EUR $\left.r^{2}=0.99\right)$ is 
predicted to be deleterious/probably damaging and could be the causal variant at the locus. The Ser37Phe variant is also in high LD $\left(r^{2}>0.8\right)$ with several nearby noncoding variants (Supplementary Fig. 7). However, none of these overlap with regulatory elements in adipocytes. The expression of ACTL9 is restricted to the testis, and it is therefore likely to act in an adipocyte-nonautonomous fashion to influence leptin concentrations. Actin proteins have cytoskeletal functions and have also been implicated in signaling and nuclear activities.

\section{Gene Set Analyses Implicate Adipocyte-Related Pathways}

We performed gene set enrichment analyses using ECDEPICT $(18,19,47)$ and PASCAL $(20)$ to identify biological processes and candidate pathways enriched for loci associated with leptin unadjusted or adjusted for BMI. Among coding variants associated with BMI-unadjusted leptin concentrations, PASCAL identified significant enrichment of the gene set for "positive regulation of reproductive success" $\left(P_{\text {empirical }}=1.6 \times 10^{-5}\right)$ (Supplementary Table 13), consistent with the crucial permissive role of leptin in the integrity of the gonadal axis (48). Among coding variants associated with leptin adjusted for BMI, we found enrichment of the immune-related TRIM39 protein-protein interaction subnetwork $(49,50)\left(P_{\text {empirical }}=8.4 \times 10^{-6}\right)$ (Supplementary Table 14). No gene sets were found to be significantly enriched in PASCAL analyses where all exome-wide variants (coding and noncoding) for leptin adjusted for BMI were included or in the EC-DEPICT analyses.

\section{DISCUSSION}

We identified 10 genetic variants associated with leptin concentrations and two gene-based associations using an exome-based genotyping array in up to 57,232 individuals with varying ancestries. The two independent variants most strongly associated with leptin concentrations were located in and near the LEP gene. The African ancestrydriven variant within LEP, Val94Met (rs17151919), was found to decrease leptin secretion in HEK293 cells, whereas rs10487505, located near $L E P$, overlaps a long noncoding (lnc)RNA that regulates LEP expression (10). Both variants showed significant association with increased adiposity in children, whereas only a nominal or no association was observed in adults.

Previous analyses have shown that the leptin-lowering allele of rs10487505 is only weakly associated with higher BMI in adulthood but shows a pronounced association with BMI in early childhood $(10,11)$. Similarly, we showed that the LEP Met94 allele, associated with lower leptin concentration, is associated with early childhood BMI. Our results suggest that leptin has an impact specifically on early adiposity, encouraging further studies to uncover the molecular mechanisms that underlie this age-dependent relationship between leptin and BMI.
The Val94Met and rs10487505 variants in and near $L E P$ are likely to influence leptin concentrations by different molecular mechanisms. The novel African ancestrydriven variant Val94Met may affect circulating levels of leptin by reducing leptin secretion. The rs10487505 variant is associated with leptin mRNA levels in adipose tissue. We hypothesize that this variant, located upstream of LEP within an lncRNA (EL947753), interacts with enhancer regions to regulate the expression of $L E P$. Defects in $L E P$ regulation in mice lead to a relative hypoleptinemic form of obesity that is responsive to leptin administration (10).

We identified four new loci associated with leptin concentrations, located in or near the ZNF800, KLF14, KLHL31, and ACTL9 genes. Two additional genes, CNTD1 and DNAJC18, were identified in gene-based analyses. The ZNF800 and KLF14 genes are master trans regulators of adipose tissue gene expression (26) and located in the proximity of the LEP gene ( $866 \mathrm{~kb}$ and $2.5 \mathrm{Mb}$ away, respectively). The variants in ZNF800 and near KLF14 were not associated with LEP mRNA levels, however, suggesting that they may be involved in translational or posttranslational rather than transcriptional regulation of leptin production. KLHL31 has been shown to promote adipocyte differentiation and suppress oxidative metabolism in adipocytes, whereas ACTL9 is not expressed in adipocytes and could affect circulating levels by a noncell-autonomous mechanism. The KLHL31 and ACTL9 loci, and the CNTD1 and DNAJC18 genes, were only identified in sex-specific models and narrowly passed the array-wide significance threshold. Further validation of the association of these loci with leptin concentrations is warranted.

In summary, we identified a new genetic association of an African ancestry-specific missense variant rs17151919 in $L E P$ with leptin concentrations and replicated the association of the rs10487505 variant near LEP. The pronounced association of these variants with BMI in early childhood implicates genetic regulation of $L E P$ in early growth and suggests that young children may be particularly sensitive to the metabolic/behavioral effects of leptin. We also identified novel loci at ZNF800, KLF14, KLHL31, ACTL9, CNTD1, and DNAJC18 associated with leptin concentrations, providing additional insights into leptin physiology.

Acknowledgments. The authors thank all investigators, study members, and study participants for their contributions to the participating studies. The acknowledgments for all participating studies are provided in the Supplementary Material. This research has been conducted using the UK Biobank resource and carried out under UK Biobank project 9072. Details on patient and public involvement in the UK Biobank are available online at www.ukbiobank.ac.uk/ about-biobank-uk and www.ukbiobank.ac.uk/wp-content/uploads/2011/07/ Summary-EGF-consultation.pdf?phpMyAdmin=trmKQIYdjnQlgJ\%2CfAzikMhEnx6. Funding and Duality of Interest. The funding information for all participating studies are provided in the Supplementary Material. D.M.-K. is a part-time clinical research consultant for Metabolon, Inc. M.A.N.'s 
participation is supported by a consulting contract between Data Tecnica International and the National Institute on Aging, National Institutes of Health. V.S. has served on advisory boards for Novo Nordisk and Sanofi and received honoraria from these companies. V.S. also has ongoing research collaboration with Bayer Ltd. B.M.P. serves on the Steering Committee of the Yale Open Data Access Project funded by Johnson \& Johnson. J.R.K. reports stock ownership in Bristol-Myers Squibb, Johnson \& Johnson, Merck, and Pfizer. R.S.F. is currently an employee of Vertex Pharmaceuticals Incorporated, though all work for this article was done prior to Vertex affiliation. This work was supported by NHGRI NIH F31HG009850. E.I. is now an employee of GlaxoSmithKline, but this work was done prior to joining the company. No other potential conflicts of interest relevant to this article were reported.

The ongoing research by V.S. in collaboration with Bayer Ltd. is unrelated to the current study.

Author Contributions. H.Y., Y.Z., R.J.F.L., and T.O.K. comprised the central analysis and writing team. R.L.-G, N.G., X.G., L.-P.L., M.F.F., A.M., T.Kac., D.I.C., N.A., L.B., N.R., K.L.Y., J.B.B., J.B.-J, P.C., A.D., M.G., Y.-D.I.C., P.J., J.K., J.Lu., S.Mä., C.M.-G., D.O.M.-K., B.M.P., R.A.S., K.S., A.T., A.G.U., Y.W., P.K., C.M.v.D., P.M.R., G.H., E.I., M.A.P., T.L., L.S.A., J.I.R., J.G.W., H.G., T.H., N.J.W., C.L., C.M.L., and K.L.M. contributed to genotyping. H.Y., Y.Z., C.N.S., T.Kar., L.O.H., J.B., C.S., R.S.F., M.H.P., Z.K., L.B.L.W., Y.U., S.M., S.M.W., R.L.-G., N.G., S.W., S.Mo., A.A.S., M.A.N., L.A.L., J.Ha., X.G., L.-P.L., M.F.F., C.M.S., C.V., A.M., T.Kac., C.A.W., D.I.C., N.A., L.B., N.R., K.L.Y., P.L.A., J.B.-J., A.D., C.A.D., K.G., H.H., H.J., J.Lu., M.M., C.M.-G., A.P.M., I.P., K.S., A.T., J.Y., H.G., K.L.M., R.L.L., and T.O.K. contributed to statistical analysis within cohorts. M.F.F., C.V., N.A., K.L.Y., M.A., M.B., J.B.B., G.D.C., A.D., M.E.G., M.G., J.Ho., R.J., P.J., A.E.J., M.K., J.R.K., C.A.L., J. Li, L.L., D.A.M., S.Mä., J.F.M.C., C.M.-G., D.O.M.-K., R.d.M., M.N., A.D.P., B.M.P., O.T.R., T.S., K.D.T., Y.W., M.A.I., C.M.v.D., M.A.P., T.L., L.S.A., J.I.R., J.G.W., H.G., J.B.M., N.J.W., T.M.F., and C.L. contributed to sample collection and phenotyping. N.G., D.I.C., R.J., M.K., L.L., M.M., C.E.P., O.T.R., K.S., A.G.U., M.W., K.E.N., M.A.I., P.M.R., S.L., E.I., T.D.S., B.M., T.L., L.S.A., J.I.R., A.P.R., T.B.H., S.R., H.G., J.B.M., V.S., T.H., K.W.v.D., N.J.W., S.F.A.G., C.L., and K.L.M. were the Principal Investigators within cohorts. H.Y. and T.O.K. are the guarantors of this work and, as such, had full access to all the data in the study and take responsibility for the integrity of the data and the accuracy of the data analysis.

\section{References}

1. Ahima RS, Prabakaran D, Mantzoros C, et al. Role of leptin in the neuroendocrine response to fasting. Nature 1996;382:250-252

2. Faroogi IS, Matarese G, Lord GM, et al. Beneficial effects of leptin on obesity, T cell hyporesponsiveness, and neuroendocrine/metabolic dysfunction of human congenital leptin deficiency. J Clin Invest 2002;110:1093-1103

3. Montague CT, Faroogi IS, Whitehead JP, et al. Congenital leptin deficiency is associated with severe early-onset obesity in humans. Nature 1997;387: 903-908

4. Farooqi IS, Keogh JM, Kamath S, et al. Partial leptin deficiency and human adiposity. Nature 2001;414:34-35

5. Wabitsch M, Funcke JB, Lennerz B, et al. Biologically inactive leptin and early-onset extreme obesity. N Engl J Med 2015;372:48-54

6. Chung WK, Belfi K, Chua M, et al. Heterozygosity for Lep(ob) or Lep(rdb) affects body composition and leptin homeostasis in adult mice. Am J Physiol 1998; 274:R985-R990

7. Narkiewicz K, Szczech R, Winnicki M, et al. Heritability of plasma leptin levels: a twin study. J Hypertens 1999;17:27-31

8. Rice T, Chagnon YC, Borecki IB, et al. Familial resemblance for plasma leptin: sample homogeneity across adiposity and ethnic groups. Obes Res 2002;10: 351-360

9. Kilpeläinen TO, Carli JF, Skowronski AA, et al. Genome-wide meta-analysis uncovers novel loci influencing circulating leptin levels. Nat Commun 2016;7: 10494
10. Dallner OS, Marinis JM, Lu YH, et al. Dysregulation of a long noncoding RNA reduces leptin leading to a leptin-responsive form of obesity. Nat Med 2019;25: 507-516

11. Helgeland $\emptyset$, Vaudel $M$, Juliusson PB, et al. Genome-wide association study reveals dynamic role of genetic variation in infant and early childhood growth. Nat Commun 2019;10:4448

12. Feng $\mathrm{S}$, Liu $\mathrm{D}$, Zhan $\mathrm{X}$, Wing MK, Abecasis GR. RAREMETAL: fast and powerful meta-analysis for rare variants. Bioinformatics 2014;30:2828-2829

13. Zhan X, Hu Y, Li B, Abecasis GR, Liu DJ. RVTESTS: an efficient and comprehensive tool for rare variant association analysis using sequence data. Bioinformatics 2016;32:1423-1426

14. Liu DJ, Peloso GM, Zhan X, et al. Meta-analysis of gene-level tests for rare variant association. Nat Genet 2014;46:200-204

15. Wu MC, Lee S, Cai T, Li Y, Boehnke M, Lin X. Rare-variant association testing for sequencing data with the sequence kernel association test. Am J Hum Genet 2011;89:82-93

16. Price AL, Kryukov GV, de Bakker PI, et al. Pooled association tests for rare variants in exon-resequencing studies. Am J Hum Genet 2010;86:832-838

17. Grant SF, Bradfield JP, Zhang H, et al. Investigation of the locus near MC4R with childhood obesity in Americans of European and African ancestry. Obesity (Silver Spring) 2009;17:1461-1465

18. Pers TH, Karjalainen JM, Chan Y, et al.; Genetic Investigation of ANthropometric Traits (GIANT) Consortium. Biological interpretation of genome-wide association studies using predicted gene functions. Nat Commun 2015;6:5890 19. Turcot V, Lu Y, Highland HM, et al.; CHD Exome+ Consortium; EPIC-CVD Consortium; ExomeBP Consortium; Global Lipids Genetic Consortium; GoT2D Genes Consortium; EPIC InterAct Consortium; INTERVAL Study; ReproGen Consortium; T2D-Genes Consortium; MAGIC Investigators; Understanding Society Scientific Group. Protein-altering variants associated with body mass index implicate pathways that control energy intake and expenditure in obesity. Nat Genet 2018;50:26-41

20. Lamparter D, Marbach D, Rueedi R, Kutalik Z, Bergmann S. Fast and rigorous computation of gene and pathway scores from SNP-based summary statistics. PLOS Comput Biol 2016;12:e1004714

21. Moayyeri A, Hammond CJ, Valdes AM, Spector TD. Cohort Profile: TwinsUK and healthy ageing twin study. Int J Epidemiol 2013;42:76-85

22. Boyd A, Golding J, Macleod J, et al. Cohort Profile: the 'children of the 90s'the index offspring of the Avon Longitudinal Study of Parents and Children. Int $\mathrm{J}$ Epidemiol 2013;42:111-127

23. Aschard $H$, Vilhjálmsson BJ, Joshi AD, Price AL, Kraft P. Adjusting for heritable covariates can bias effect estimates in genome-wide association studies. Am J Hum Genet 2015;96:329-339

24. Cole SR, Platt RW, Schisterman EF, et al. Illustrating bias due to conditioning on a collider. Int J Epidemiol 2010;39:417-420

25. Yaghootkar H, Bancks MP, Jones SE, et al. Quantifying the extent to which index event biases influence large genetic association studies. Hum Mol Genet 2017;26:1018-1030

26. Civelek M, Wu Y, Pan C, et al. Genetic regulation of adipose gene expression and cardio-metabolic traits. Am J Hum Genet 2017;100:428-443

27. Lee MJ, Wu Y, Fried SK. A modified protocol to maximize differentiation of human preadipocytes and improve metabolic phenotypes. Obesity (Silver Spring) 2012;20:2334-2340

28. Pettersen EF, Goddard TD, Huang CC, et al. UCSF Chimera-a visualization system for exploratory research and analysis. J Comput Chem 2004;25: $1605-1612$

29. Dunbrack RL Jr. Rotamer libraries in the 21 st century. Curr Opin Struct Biol 2002;12:431-440

30. Pandurangan AP, Ochoa-Montaño B, Ascher DB, Blundell TL. SDM: a server for predicting effects of mutations on protein stability. Nucleic Acids Res 2017;45: W229-W235

31. Chen CW, Lin J, Chu YW. iStable: off-the-shelf predictor integration for predicting protein stability changes. BMC Bioinformatics 2013;14(Suppl. 2):S5 
32. Parthiban V, Gromiha MM, Schomburg D. CUPSAT: prediction of protein stability upon point mutations. Nucleic Acids Res 2006;34:W239-W242

33. Capriotti E, Calabrese R, Casadio R. Predicting the insurgence of human genetic diseases associated to single point protein mutations with support vector machines and evolutionary information. Bioinformatics 2006;22:2729-2734

34. Zhang M, Guller S, Huang Y. Method to enhance transfection efficiency of cell lines and placental fibroblasts. Placenta 2007;28:779-782

35. Karczewski KK, Francioli LC, Tiao G,et al. Variation across 141,456 human exomes and genomes reveals the spectrum of loss-of-function intolerance across human protein-coding genes. 30 January 2019 [preprint]. bioRxiv: 531210

36. Friedlander Y, Li G, Fornage M, et al. Candidate molecular pathway genes related to appetite regulatory neural network, adipocyte homeostasis and obesity: results from the CARDIA Study. Ann Hum Genet 2010;74:387-398

37. Ng MCY, Graff M, Lu Y, et al.; Bone Mineral Density in Childhood Study (BMDCS) Group. Discovery and fine-mapping of adiposity loci using high density imputation of genome-wide association studies in individuals of African ancestry: African Ancestry Anthropometry Genetics Consortium. PLoS Genet 2017; 13:e1006719

38. Najafabadi HS, Mnaimneh S, Schmitges FW, et al. C2H2 zinc finger proteins greatly expand the human regulatory lexicon. Nat Biotechnol 2015;33: $555-562$

39. Hernandez Cordero Al, Gonzales NM, Parker CC, et al. Genome-wide associations reveal human-mouse genetic convergence and modifiers of myogenesis, CPNE1 and STC2 [published correction appears in Am J Hum Genet 2020; 106:138]. Am J Hum Genet 2019;105:1222-1236

40. Voight BF, Scott LJ, Steinthorsdottir V, et al.; MAGIC investigators; GIANT Consortium. Twelve type 2 diabetes susceptibility loci identified through largescale association analysis. Nat Genet 2010;42:579-589

41. Lotta LA, Gulati P, Day FR, et al.; EPIC-InterAct Consortium; Cambridge FPLD1 Consortium. Integrative genomic analysis implicates limited peripheral adipose storage capacity in the pathogenesis of human insulin resistance. Nat Genet 2017; 49:17-26

42. Teslovich TM, Musunuru K, Smith AV, et al. Biological, clinical and population relevance of 95 loci for blood lipids. Nature 2010;466:707-713

43. Shungin D, Winkler TW, Croteau-Chonka DC, et al.; ADIPOGen Consortium; CARDIOGRAMplusC4D Consortium; CKDGen Consortium; GEFOS Consortium; GENIE Consortium; GLGC; ICBP; International Endogene Consortium; LifeLines Cohort Study; MAGIC Investigators; MuTHER Consortium; PAGE Consortium; ReproGen Consortium. New genetic loci link adipose and insulin biology to body fat distribution. Nature 2015;518:187-196

44. Small KS, Todorčević M, Civelek M, et al. Regulatory variants at KLF14 influence type 2 diabetes risk via a female-specific effect on adipocyte size and body composition. Nat Genet 2018;50:572-580

45. $\mathrm{Ng} \mathrm{PC}$, Henikoff S. SIFT: predicting amino acid changes that affect protein function. Nucleic Acids Res 2003;31:3812-3814

46. Adzhubei I, Jordan DM, Sunyaev SR. Predicting functional effect of human missense mutations using PolyPhen-2. Curr Protoc Hum Genet 2013;Chapter 7: Unit7.20

47. Marouli E, Graff M, Medina-Gomez C, et al.; EPIC-InterAct Consortium; CHD Exome + Consortium; ExomeBP Consortium; T2D-Genes Consortium; GoT2D Genes Consortium; Global Lipids Genetics Consortium; ReproGen Consortium; MAGIC Investigators. Rare and low-frequency coding variants alter human adult height. Nature 2017;542:186-190

48. Rosenbaum M, Leibel RL. Leptin: a molecule integrating somatic energy stores, energy expenditure and fertility. Trends Endocrinol Metab 1998;9:117-124 49. Kurata R, Nakaoka H, Tajima A, et al. TRIM39 and RNF39 are associated with Behçet's disease independently of HLA-B*51 and $-A^{\star} 26$. Biochem Biophys Res Commun 2010;401:533-537

50. Suzuki M, Watanabe M, Nakamaru $Y$, et al. TRIM39 negatively regulates the $\mathrm{NF}_{\kappa} \mathrm{B}$-mediated signaling pathway through stabilization of Cactin. Cell Mol Life Sci 2016;73:1085-1101 